\title{
SOME ASPECTS OF WIRELESS SENSOR NETWORKS
}

\author{
Yogesh Kumar Fulara \\ Department of Electronics and Communication Engineering, \\ PEC University of Technology, Chandigarh, India
}

\begin{abstract}
The development of the wireless sensor networks (WSNs) in various applications like Defense, Health, Environment monitoring, Industry etc. always attract many researchers in this field. WSN is the network which consists of collection of tiny devices called sensor nodes. Sensor node typically combines wireless radio transmitter-receiver and limited energy, restricted computational processing capacity and communication band width. These sensor node sense some physical phenomenon using different transduces. The current improvement in sensor technology has made possible WSNs that have wide and varied applications. While selecting the right sensor for application a number of characteristics are important. This paper provides the basics of WSNs including the node characteristics. It also throws light on the different routing protocols.
\end{abstract}

\section{KEYWORDS}

Communication capability, Routing Protocols, Sensor node, WSNs.

\section{INTRODUCTION}

WSNs were primarily introduced for the defense application and the objective here is to monitor activity of enemy without any human interference. A low-flying airplane, ground vehicle or a powerful laptop acted as a base station to collect information from all sensor nodes.

WSNs can be presume as a special case of Ad Hoc networks. WSN are generally assumed to be energy restrained because of tiny size sensor node. Even though sensor networks are a subset of Ad Hoc Network, the protocol designed for the Ad Hoc networks cannot be used as it due to the following reason:

a. The number of sensor nodes in a sensor network is very large compared to the Ad Hoc network. Thus sensor networks require distinct and more adaptable solutions.

b. As compared to Ad Hoc network, Wireless sensor nodes have restricted power supply. And in impractical environment condition they cannot be recharged because the large number of sensor nodes are deployed in different locations. 


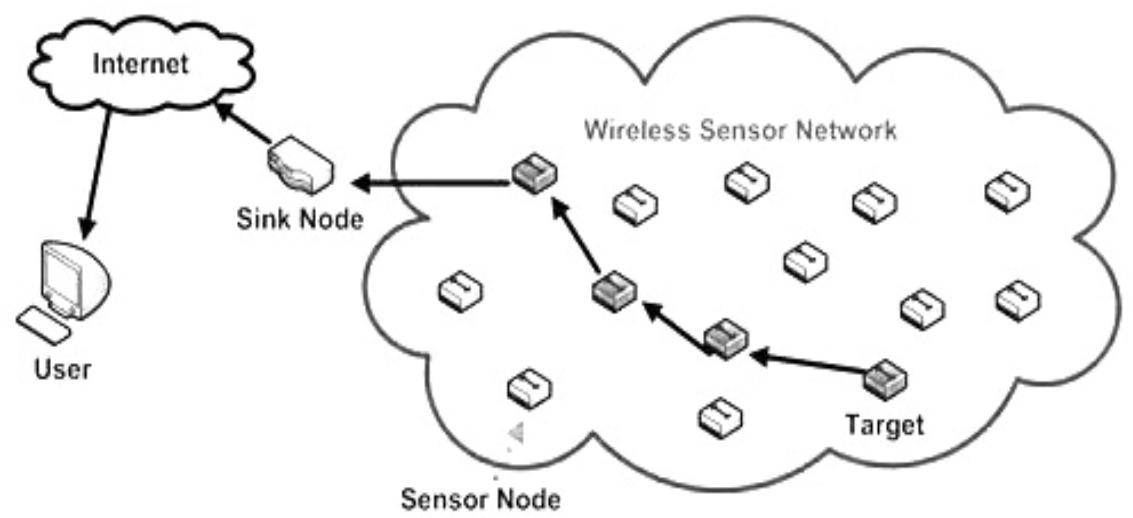

Figure: 1 Wireless Sensor Networks (WSNs)

Thus wireless sensor networks can be defined as "A WSNs consists of spatially distributed autonomous sensor to monitor physical or environmental conditions (i.e. temperature, humidity, pollution, sound, pressure, etc.) and to cooperatively pass their data through the network to user location". The WSNs is built of few to several thousands of sensors of nodes, where each and every node is connected to one or many sensor nodes. Source node is connected to a central gateway, also called as base station. Central gateway provides a connection to world through different communication channels (internet, Wi-Fi, WI-Max, wired LAN etc.). Figure: 1 shows data collection from different nodes which further processed and analyzed.

\section{COMPONENTS OF WSNS}

\subsection{Sensor Node}

Sensor nodes are generally consist of few sensors and processing unit/mote as shown in figure: 2 . a sensor is device which senses the information and pass it on to mote. Sensor are typically used to measure the change in physical environmental parameters like temperature, pressure, humidity, sound and change in the health parameter of person e.g. heartbeat and blood pressure.

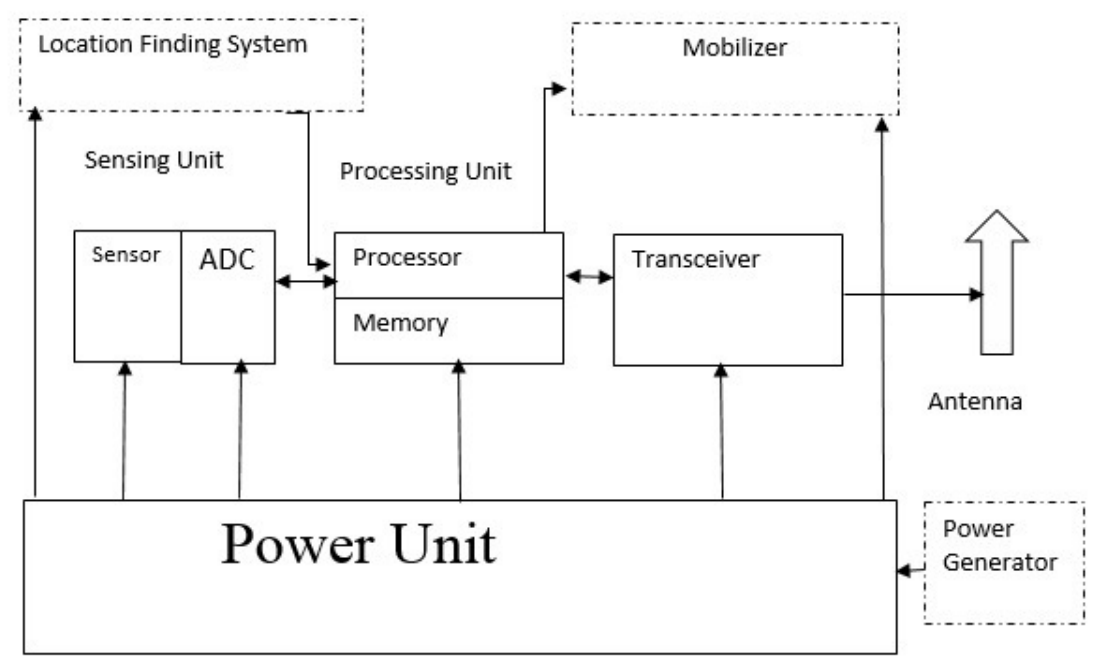

Figure: 2 Block diagram sensor networks 


\subsection{Base Station}

Different wireless sensor network are connected with base station. It consists of a microprocessor, antenna, radio board and USB interface board. For communication with wireless sensor nodes, Base station is preprogramed with low-power mesh networking software. As all the sensor nodes handover their data to base station so it is very important to deploy base station in wireless sensor network. Further this data is analysed at base station for processing and decision making. During deployment of base station in sensor network coverage of sensor nodes, Energy conservation and reliability issues are taken care of. Generally base stations are assumed static in nature but in some scenarios, they are assumed to be mobile to collect data from sensor nodes.

\section{WSNS REQUIREMENTS AND CHARACTERISTICS}

Tiny size, limited power supply, specific application and other various constraints make Wireless sensor networks different from general wireless networks. The system performance characteristics varies in WSNs even though the same basic principles of wireless communication network like size, power, cost and their fundamental constraints in WSNs. Considering the basic difference with the wireless system, major points which affect the performances and design of WSNs are the following:

\subsection{Deployment}

The deployment strategy depends mainly on the type of sensor and the application.

\subsection{Localization Scheme}

Localization is the method to find the accurate physical position of sensor nodes.

\subsection{Scalability}

It is the ability of the network to grow without excessive overhead.

\subsection{Reliability}

The ability of network for authentic data transmission while continuous change of network structure.

\subsection{Responsiveness}

It is the ability of the network to quickly accommodate itself to change in topology.

\subsection{Mobility}

It is the ability of the network to maintain mobile nodes and unstable data paths.

\subsection{Quality of service implementation}

In some applications (e.g. military application) data should be delivered within the certain period of time from the moment it is sensed, otherwise the data will be useless. 


\subsection{Security}

Security is an important issue which implies that both authentication \& encryption should be feasible.

\subsection{Characteristics of WSNs}

Wireless sensor networks are strongly restricted by energy, capacity and computing power. So it is essential to design effective network in order to increase the network characteristics. The important characteristics of WSNs are as follows:

- Minimum Power consumption

- Ability to cope with node failures (resilience)

- Heterogeneity of nodes

- Mobility of nodes

- Communication failure

- Scalability to large scale of deployment

- Ability to withstand in unfavorable environmental conditions

- Cross-layer design

- Ease of use

\section{ROUTING PROTOCOLS IN WIRELESS SENSOR NETWORKS}

WSNs is the networks in which the sensor node are limited to various resources itself, so the main objective is to design an efficient and energy sensible protocol in order to prolong the network lifetime for specific application. Every sensor node are not defined with the unique ID, there are lot of redundant data collected at destination nodes. So while designing the routing protocols for wireless sensor networks we must keep in mind the following parameter like energy efficiency, scalability, accuracy, fault-tolerance, latency and QOS. Generally most of the routing protocol are classified as:

\subsection{Data-centric Routing protocols}

In this routing protocol, the node send queries to surrounding regions and waits for data from the sensor located in the selected regions. Since data is being requested through queries, attribute based naming is necessary to specify the properties of data. In this routing protocol data is frequently transmitted from every senor node within the deployment region.

The following protocols are discussed in this category:

\section{Flooding and Gossiping:}

These are the most traditional network routing. They do not require to know the network topology or any routing algorithms. In flooding mechanism, each and every sensor node receives a data and then transmits it to all neighboring nodes. Gossiping is somewhat enhanced version of flooding in which receiving node sends the packet to randomly selected neighbors, which select another random neighbor to process or forward the packet to and so on [1]. 


\section{Directed Diffusion:}

All the nodes in directed diffusion based network are application-aware. This enables diffusion to achieve energy saving. In directed diffusion, base station and sensor create gradient of information in their respected neighborhoods. The base station request data by broadcasting query and interest is diffused through the network hop-by-hop, and is broadcast by each neighbors. As the interest is propagated everywhere in the network, gradients are setup to draw path satisfying shortest path from each node towards base station. This process continues until gradients are setup from each node back to the base station [2].

\section{SPIN:}

Joanna Kulik et al. in [1] , [3] proposed a family of adaptive protocol, called SPIN (Sensor Protocol for Information via Negotiation) that efficiently expand data among sensor nodes in an energy-constrained WSNs and overcome the problem of implosion and overlap occurred in classic flooding. Nodes running a SPIN communication protocol name their data using high-level data descriptors, called metadata. SPIN nodes negotiate with each other before transmitting data. Negotiation helps to ensure that the transmission of redundant data throughout the network is eliminated and only useful information will be transferred.

\section{Gradient-base routing:}

This routing algorithm makes an improvement on direct Diffusion, in order to get the total minimum hop other than the total shortest time and also aims to establish the cost field and minimum cost [4].

\subsection{Hierarchical-Based Routing}

Hierarchical routing provides an approach for point to point routing with very small routing state. Scalability is one of the essential design features of sensor network. Cluster or Hierarchical based Routing methods are well known techniques with special advantage of efficient communication and scalability. Hierarchical routing maintain the energy consumption of senor nodes and performs data aggregation which help in decreasing the number of transmitted message to base station. Some protocol are discussed here

\section{LEACH:}

LEACH stand for Low-Energy Adaptive Clustering Hierarchy and is one of the first hierarchical protocols. It is self-organizing, adaptive clustering protocol in which sensor nodes will organize themselves into local cluster and cluster members elect cluster head to avoid excessive energy consumption and incorporate data aggregation which reduced the amount of message sent to the base station, to increase the life time of the network [5].

\section{TEEN and APTEEN:}

TEEN stands for threshold sensitive Energy Efficient sensor network protocol. In TEEN, sensor networks sense their environment continuously, thereby making it appropriate for real time applications. TEEN was the first protocol developed for reactive networks and used in temperature sensing application which reduces the energy consumption in network [6]. The Adaptive Periodic TEEN (APTEEN) is an enhancement over TEEN protocol which is able to combine the best features of proactive and reactive network while minimizing their limitations to 
create a hybrid network. In this scheme, only periodic data send by the sensor network, they also acknowledge to sudden changes in attribute values [7].

\subsection{Location Based Routing}

Most of the routing protocol require location information for sensor nodes in wireless sensor network to calculate the distance between two particular nodes on the basis of signal strength so that the energy consumption can be estimate. If we don't know the addressing scheme for sensor network then we can also apply the location based routing for sensor data in energy efficient way. Some of them are discussed here as:

\section{MECN and SMECN:}

Minimum energy communication network sets up and maintains a minimum energy network for wireless networks by utilizing low power GPS. Although, the protocol assumes a mobile network, it is best applicable to sensor network, which are not mobile [8]. The small minimum energy Communication network (SMECN) is an improved version of MECN. It assumed that every node can transmit to every other node, which is not possible every time [9].

\section{GEAR (Geographic and Energy Aware Routing):}

The objective of GEAR is to minimize the number of interest in direct diffusion routing and add geographic data into interest packet by only assuming a certain region rather than sending interest the whole network. GEAR help in balancing energy consumption in this way and increase the life time [10].

\section{Fermat Point Based Energy Efficient Geocast Routing:}

Within a specified geographical area (called Geocast region) all nodes are delivered by Geocast routing protocol. Fermat point based protocol are adapted for reducing the energy consumption of a WSN by reducing the total transmission distance in a multi hop multi sink scenario [11].

\section{ApPLication OF WSNS}

\subsection{Security and surveillance}

Security and detection are the important application of wireless sensor networks. Sensor node with motion capabilities may be deployed at the borders to detect the intruder crossing the line of control. Hence surveillance of regions, assets, perimeter, borders and cleared areas can be efficiently done by deploying wireless sensor networks.

\subsection{Environmental monitoring}

The term Environmental Sensor Networks have evolved to cover many applications of WSNs to earth science research including sensing volcanoes, oceans, glaciers, forests etc. Some examples of major areas listed below.

\section{Air quality/Air pollution monitoring}

Wireless sensor network have been deployed in several cities to monitor the concentration of dangerous or harmful gases for human. For such applications there are distinct architectures and different kinds of data analysis and data mining. 


\section{Water quality monitoring}

The distribution of wireless sensors enables the creation of a more accurate map of the water status, and allows the permanent deployment of monitoring stations in locations of difficult access, without the need of manual data retrieval.

\section{Natural disaster monitoring}

It is used to prevent the consequences of natural disasters, like floods.

\section{Forest fire detection}

To detect the when fire has started in forest, a network of Sensor Nodes can be installed. With the help of such type of wireless sensor networks we can take early action to protect forest.

\section{Landslide detection}

With the help of wireless sensor network we can forecast earthquake or landslide by detect the slight movements and various parameter changes of soil that may occur before or during it happen.

\section{Habitat monitoring}

Habitat monitoring is one of the essential parts in environmental monitoring. Habitat means a place in which an animal or plant naturally grows or lives. Therefore, habitat monitoring is an important to make sure their species autonomies and prevent any ecological disturbance for animals and plants. Through WSNs, habitat monitoring can be enhance and protect the life of animals and plants.

\section{Climate monitoring}

The climate change of the world nowadays have brought many effects such as the breaking of sea ice, increasing in sea water level, heat wave, glacier melting, temperature warming and many more. Thus using WSNs we can monitor and can try to minimize these harms.

\subsection{Building, Bridge and Structural monitoring}

Several recent projects have explored the use of sensor network in monitoring the health of building, bridge and highway. Fiber optic based, Bluetooth, etc. wireless sensor network are used to monitor stress, vibration, temperature and crack opening due to strain and corrosion of the reinforcement in concrete bridge decks and structures. The use of sensor network for controlling civil and mechanical system, has been explored in by having acquisition, monitoring and recording of data, detecting failure, monitoring state and making control decisions.

\subsection{Industrial monitoring system}

WSNs have been used for machinery condition-based maintenance as they offer significant cost saving and enable new functionality. The new aspects are considered as an enabler for future application in industrial and related wireless sense and control application and partially replacing or enhancing conventional wire-based network by WSN techniques. 


\subsection{Agriculture}

Wireless network frees the farmer from the maintenance of wiring in an unfavorable environment. Under water system can be monitored using pressure transmitters to detect tank level and pump can be controlled using wireless devices and water use can be measured and wirelessly transmitted back to a center for billing.

\subsection{Health Care Monitoring}

WSNs can enhance the human health monitoring system. In this category include tele monitoring of human of human health is possible. We can monitored physiological data and we can monitoring of doctor and patients inside a hospital, drug administrator in hospitals and so on.

\subsection{Greenhouse monitoring}

Nowadays, we witness more and more electronic application in an average household. Therefore, great commercial opportunities exist for home automation and smart home/office environment cooling, heating and humidity control. These parameters can be monitored and controlled by WSNs.

\section{LiTERATURE SURVEY}

R. Yan [senior Member IEEE], H. sun and Y. Qian, "Energy-Aware Sensor Node Design with its application in wireless sensor network". In this paper, they present the design and implementation of energy-aware sensor node, which can help in constructing energy-efficient WSNs by calculating distance between nodes, periodic sleep/wake-up scheme for data transmission. Furthermore they discussed energy saving can be achieved by different network configuration and choosing the most energy efficient one [12].

HE Bin, ZHANG Hongtao, "An energy optimization method for wireless sensor Network". In this paper research was done, how the energy optimized in the WSNs. They used the Dijkstra shortage path algorithm to calculate the optimal sequence of transmission distance from source node and sink node. There q-switch algorithm was used to keep balance of network by choosing one with maximum energy within each q type of nodes. [13].

Sukun Kim, Rodrigo Fonseca and David Culler, "Reliable transfer on wireless sensor network." In this paper, different types of routing are proposed. Link level transmission, erasable code and route fix were used and calculated. Erasable code introduces static overhead. Route fix reduces consecutive losses, increasing usefulness of erasable [14]

Stephan H. Chagas, Joao B. Martins. "An Approach to localization Scheme of wireless sensor network based on artificial Neural Network and Genetic Algorithms." This paper presented an approach to localization scheme for WSNs using artificial neural network as the machine learning algorithm and genetic algorithms to select the best ANN structure [15].

N. Javaid, T.N. Khan, A. Iqbal, A. Akhtar, M. Ishfaq, "Enhanced Developed Distributed Energy-Efficient Clustering for Heterogeneous Wireless Sensor Networks." In this paper, they proposed a novel clustering based routing technique for heterogeneous WSNs. The technique was based on changing dynamically and with more efficiency cluster Head election probability [16].

Hongyang Chen, Kaoru Swzaki, Ping Deng and Hing Cheung so, "An improved DV-Hop localization Algorithm for Wireless Sensor Networks" In this paper they develop new method, 
Improved DV-Hop algorithm significantly. The simulation result showed that the suggested algorithm can improve accurate location and coverage than DV-Hop algorithm. This approach is effective and surely has good application foreground [17]

Quazi Mamum, Mohammed Kaosar, "What is the first step in designing an Application Protocol for wireless sensor Networks." This paper present a novel paradigm in designing application protocol for WSNs. In this paper they determined how a well-designed logical topology effect the performances of protocols developed in WSNs. By this, the logical structure and the communication abstraction of the logical topology are used to design a number of application protocols and their performance can evaluated [18].

V. M. Priyadarshini, N. Muthukumar and M. Natarajan, "Cellular Architecture Sensors for Wireless Sensor Networks." In this paper, they have worked on WSNs nodes and arrange nodes to improve coverage area, reliability in getting information from the nodes. They also worked on minimizing loss of information. This architecture arrangement provides recent solution to security and military applications [19].

Md. Abdul Alim, Yucheng Wu, Wei Wang," A Fuzzy Base Clustering Protocol for EnergyEfficient Wireless Sensor Networks." In this paper, a fuzzy logic based energy-aware dynamic clustering technique is proposed, which increases the network life time in terms of last node dies. Through this protocol they optimized the number of cluster formed in every round, which is not possible in LEACH. They demonstrates that this approach is better than LEACH in terms of energy saving as well as network life time [20].

\section{CONCLUSION AND FUTURE DIRECTIONS}

WSN is one of the fastest growing areas in the broad wireless Ad Hoc networking field. The research in WSNs is thriving at a rapid pace and is being studied as the revolutionary concept of current era. But, still there are many challenges that need to be explain such as, how to reduce the power source and self-power generating technology to provide indefinite power sources.Researcher's need to work on to provide secured communication with limited resource requirements. We have fractional answers and or roadmap to some above question, there is still much to be done in wireless sensor networks.

\section{REFERENCES}

[1] Joanna Kulik, HariBalakrishnan and W. R. Heinzelman, (1999) “Adaptive protocols for Information Dissemination in Wireless sensor networks", proceeding on 5th annual ACM/IEEE International conference on mobile Computing and networking, pp. 174-185.

[2] ChalermekIntanagonwiwat, Ramesh Govindan, Deborah Estrin, John Heidemann and Fabio Silva, "Directed Diffusion for Wireless Sensor Networking", IEEE/ACM Transactions on networking (TON), vol. 11, pp. 2-16, February 2003.

[3] Joanna Kulik, HariBalakrishnan and W. R. Heinzelman, "Negotiation-Based Protocols for Disseminating Information in Wireless Sensor Network," Wireless Network, vol. 8, pp. 169-185, 2002.

[4] Li Xi Chen and Xiaohong Guan, (2004) “A New Gradient-Based Routing Protocol in Wireless Sensor Networks", proceedings of the First International Conference on Embedded Software and Systems, pp. 318-325.

[5] Ankita joshi and Lakshmi Priya.m, "A survey of Hierarchical Routing Protocols in Wireless Sensor Network", MES Journal of technology and Management, pp.67-71

[6] Arati Manjeshwar and Dharma P. Agrawal, "TEEN: A Routing Protocol for Enhanced efficiency in Wireless Sensor Networks", Parallel and Distributed Processing Symposium, Proceedings, 15th International, pp 2009-2015, April. 2009. 
[7] Arati Manjeshwar and Dharma P. Agrawal, "APTEEN: A Hybrid Protocol for Efficient Routing and Comprehensive Information Retrieval in wireless Sensor Networks" Parallel and Distributed Processing Symposium, proceeding International, IPDPS 2002, Abstracts and CD-ROM, pp. 195-202.

[8] V. Rodoplu, T.H. Ming, "Minimum Energy Mobile Wireless Networks", IEEE journal of selected areas in communications Aug 17, 1999, pp. 1333-1344.

[9] L. Li, J. Y Halpern, "Minimum Energy Mobile wireless Network Revisited", Proceedings of IEEE International Conference on Communications (ICC_01), Helsinki, Finland, June 2001.

[10] Yan Yu and Ramesh Govinda, "Geographical and Energy Aware Routing Recursive Data Dissemination Protocol for Wireless Sensor Networks", 2001

[11] Kaushik Ghosh, Partha Pratim Bhattacharya and P.K.Das, Effect of Multipath Fading and PropogationEnvironment on the Performances of a Fermat Point Based Energy Efficient Geocast Routing Protocol", International Journal of Wireless \& mobile Networks, Vol. 4, No. 1, Feb 2012

[12] Ruqiang Yan(Senior Member IEEE), Hanghang Sun and Yuning, "Energy-Aware Sensor Node Design With Its Application in Wireless Sensor Networks" proceeding IEEE transactions on Instrumentation and Measurement, Vol. 62, No. 5, pp. 1183-1191, 5 May 2013.

[13] HE Bin, ZHANG Hongtao, "An energy optimization method for wireless sensor Network" IEEE, Published in Automatic Control and Artificial Intelligence (ACAI 2012), 3-5 March 2012, pp. $402-$ 406.

[14] Sukun Kim, Rodrigo Fonseca, and David Culler, "Reliable Transfer in Wireless Sensor Networks" The First IEEE International Conference on Sensor and Ad hoc Communications and Networks, Oct 2004.

[15] Stephan H. Chagas, Joao B. Martins. "An Approach to localization Scheme of wireless sensor network based on artificial Neural Network and Genetic Algorithms" published in: New Circuits and Systems Conference (NEWCAS), 2012 IEEE 10th Conference, 17-20 June 2012, pp. 137-140

[16] N. Javaid, T.N. Khan, A. Iqbal, A. Akhtar, M. Ishfaq, "Enhanced Developed Distributed EnergyEfficient Clustering for Heterogeneous Wireless Sensor Networks." Published by Elsevir B.V., on International Workshop on Body Area Networks (BASNet-2013), Procedia Computer Science 19 (2013), pp.914-919.

[17] Hongyang Chen, Kaoru Sezaki, Ping Deng, Hing Cheung So, "An Improved DV-Hop Localization Algorithm for Wireless Sensor Networks" IEEE, Vol.8, 2008.

[18] Quazi Mamum, Mohammed Kaosar, "What is the first step in designing an Application Protocol for wireless sensor Networks.”, Published in: Sensors Application Symposium (SAS, 2014 IEEE, Date of Conference: 18-20 Feb. 2014, pp. 333-338

[19] V.M.Priyadharshini, N.Muthukumar, M.Natarajan, "Cellular Architecture Sensors for Wireless Sensor Networks" IJRRSE, Vol.01 No.02, pp 47-51 June 2011.

[20] Md. Abdul Alim, Yucheng Wu, Wei Wang," A Fuzzy Base Clustering Protocol for Energy-Efficient Wireless Sensor Networks." Proceeding of the 2nd International Conference on Computer Science and Electronics Engineering (ICCSEE 2013), pp. 2874-2878.

\section{Authors}

Yogesh Kumar Fulara

PEC University of Technology, Chandigarh.

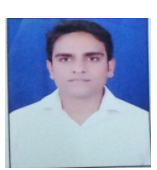

